\title{
Nonequilibrium Modeling of Oxygen in Reflected Shock Tube Flows
}

\author{
Kevin Neitzel ${ }^{1}$, Jae Gang Kim² ${ }^{2}$ and Iain D. Boyd ${ }^{3}$ \\ University of Michigan, Ann Arbor, MI 48109, USA
}

\begin{abstract}
The nonequilibrium modeling of reflected shock tube flows is investigated, motivated by hypersonic vehicle design. Oxygen nonequilibrium behavior is the focus of the work due to its contribution to modeling uncertainty, specifically the vibrational-translational energy transfer process of the $\mathrm{O}_{2}$-Ar system. Two levels of vibrational nonequilibrium modeling fidelity are evaluated. The lower fidelity model is the two-temperature model that uses Millikan-White vibration relaxation rates to capture the vibrational nonequilibrium process at the macroscopic level. The higher fidelity model is the state-resolved master equation method that uses vibrational state-to-state rates to explicitly calculate the vibrational state distribution throughout the analysis. The vibrational state-to-state rates are evaluated using the forced harmonic oscillator (FHO) model and a detailed quasi-classical trajectory (QCT) analysis. The nonequilibrium models are implemented in two flow solvers to analyze reflected shock tube experiments. First, a simple method is employed of chaining two post-normal shock analyses together to simulate the vibrational nonequilibrium behavior of a particular parcel of fluid in the reflected shock tube. Second, the nonequilibrium models are implemented in a 1-D unsteady flow solver to capture the entire behavior of the reflected shock tube. Comparisons are provided between results obtained with the two different flow solvers, and the three different physical models, for two different shock tube conditions.
\end{abstract}

\section{Nomenclature}

$\begin{array}{ll}d t & =\text { time step } \\ d x & =\text { grid spacing } \\ e & =\text { specific energy } \\ E & =\text { total energy } \\ E_{t} & =\text { translational energy } \\ E_{r} & =\text { rotational energy } \\ E_{v} & =\text { vibrational energy } \\ E_{v}{ }^{*} & =\text { equilibrium vibrational energy } \\ h & =\text { specific enthalpy } \\ H & =\text { total enthalpy } \\ i & =\text { space index } \\ n & =\text { time index } \\ p & =\text { pressure } \\ \rho & =\text { density } \\ T_{a} & =\text { geometrically-averaged temperature }\left(\sqrt{T_{t} T_{v i b}}\right) \\ T_{t} & =\text { translational temperature } \\ T_{r o t} & =\text { rotational temperature } \\ T_{v i b} & =\text { vibrational temperature } \\ \tau_{V} & =\text { vibrational relaxation time }\end{array}$

\footnotetext{
${ }^{1} \mathrm{PhD}$ Candidate, Department of Aerospace Engineering, 1320 Beal Avenue, Member of AIAA.

${ }^{2}$ Post-Doctoral Research Fellow, Department of Aerospace Engineering, 1320 Beal Avenue, Member of AIAA.

${ }^{3}$ James E. Knott Professor, Department of Aerospace Engineering, 1320 Beal Avenue, AIAA Fellow.
} 


\section{Introduction}

$\mathrm{T}$ HE development of hypersonic vehicles presents some unique issues in terms of system design. Knowledge of the flow field and physics is needed to adequately develop the thermal protection system (TPS) and control system. Due to the high enthalpy flow experienced by a hypersonic vehicle, experimental development is expensive and difficult, if not impossible. This leads to a strong reliance on computational modeling for design development. The flight conditions and geometry do not always give adequate time or distance for the flow to reach equilibrium before interacting with the vehicle. The chemistry and flow properties affecting the vehicle are governed by nonequilibrium behavior, and thus, nonequilibrium modeling is especially important. Computational modeling uncertainty has a large effect on the design process. Appropriate design margins are required in order to compensate for the modeling uncertainty.

Previous hypersonic vehicles, such as Apollo and the space shuttle, were primarily concerned with re-entry TPS design. The strong flow conditions of re-entry into the Earth's atmosphere quickly dissociate the $\mathrm{O}_{2}$ molecules in the air. Current and future hypersonic vehicles are subjected to sustained flight conditions that are milder than re-entry. The $\mathrm{O}_{2}$ molecules will not quickly dissociate and will play a large role in the flow field behavior. The development of nonequilibrium models of $\mathrm{O}_{2}$ and its interactions with the other species is crucial for limiting modeling uncertainty. The presented work will focus, specifically on, the $\mathrm{O}_{2}$ - $\mathrm{Ar}$ interaction. By focusing on highly diluted $\mathrm{O}_{2}$ cases (primarily Ar), the vibrational-translational nonequilibrium transfer process can be isolated and studied.

The standard two-temperature model represents the current state-of-the-art for nonequilibrium modeling for hypersonic vehicle design ${ }^{1,2}$. This methodology does not explicitly resolve the vibrational state populations, but captures the vibrational nonequilibrium process through macroscopic vibrational relaxation times. In particular, this method utilizes the empirically based Millikan-White vibrational relaxation times. Inherent in the application of this macroscopic approach are the assumptions that the vibrational states follow a Boltzmann distribution and that only single quantum transitions are permissible. It has been shown by previous studies that this methodology is accurate for mild, simple conditions. However, it loses applicability in stronger flow conditions where vibrational state distributions can become non-Boltzmann and multi-quantum transitions are present ${ }^{5}$. Many corrections have been proposed and implemented to improve the accuracy of the model ${ }^{1}$. These corrections however are also highly empirical and do not address the fundamental physics involved in the problem. Higher fidelity, state-to-state modeling, specifically for the vibrational energy mode, has been shown to more accurately capture the nonequilibrium physics present in stronger hypersonic flows ${ }^{3}$. The method does not assume a form of the vibrational state distribution and allows for multi-quantum transitions. State resolved modeling requires the solution of the master equation that is more computationally expensive. Additionally, obtaining state-to-state transition rates is not trivial. The current study will investigate two sets of state-to-state rates. First, the analytically based, forced harmonic oscillator (FHO) model will be used ${ }^{5,6}$. The FHO model is a widely used method due to its relatively low computational cost. Second, stateto-state rates obtained through quasi-classical trajectory (QCT) analysis are applied. QCT analysis is a high fidelity method that follows individual collision events using a potential energy surface to evaluate transition cross sections that are subsequently integrated to derive transition rates. This method represents the highest fidelity approach investigated in this study. However, QCT analysis is computationally expensive, requiring about one month of CPU time for the evaluation of the rates employed in this study.

One of the approaches for developing physical understanding of high-temperature kinetics is the shock tube experiment. A shock tube is a relatively simple method for creating high enthalpy flow conditions. Shock tube experiments allow for thermochemical nonequilibrium behavior to be observed in an effective and isolated manner. The presented work will focus on two methods of modeling the flow within a reflected shock tube. First, two steady shock analyses will be chained together to represent the incident shock and reflected shock that occur in the reflected shock tube. This method assumes perfectly uniform flow regions. Second, a fully unsteady, 1-D flow computational fluid dynamics (CFD) solver will be used to model the flow. The unsteady solver does not assume uniformity in the flow regions. One of the goals of this study will be to evaluate the importance of this potential non-uniformity on the overall solution.

In summary, the presented work will focus on two nonequilibrium modeling approaches (standard two-temperature model and the state-resolved method) and two flow modeling approaches (two-steady shock analyses and 1-D unsteady flow solver). First, a description and nomenclature is provided for reflected shock tube flows. Next, the details of the modeling approaches and experimental data are described. Finally, two test cases are then used to compare the various modeling techniques and the experimental data in order to determine requirement of fidelity in nonequilibrium modeling.

American Institute of Aeronautics and Astronautics 


\section{Shock Tube Flow}

The reflected shock tube is a simple experiment in concept, however, it is able to provide a test bed for high enthalpy, nonequilibrium flow physics. As shown in Figure 1, the shock tube is a tube with a diaphragm separating a high pressure region and a low pressure region; both volumes of fluid are initially at rest. The diaphragm is then ruptured and sets off the system of waves shown notionally in Figure 1. Regions 1, 2, and 5 are of particular interest for high enthalpy flow. The initial shock wave, known as the incident shock, produces a rise in pressure, density, and temperature from Region 1 to 2, according to the unsteady shock relations (Rankine-Hugoniot). A similar process for the reflected wave results in high enthalpy conditions in Region 5 that is consistent with the subsonic conditions downstream of the shock wave in the hypersonic flow regime. Thus, the fundamental physics of hypersonic, nonequilibrium flow can be observed with the shock tube experimental setup.

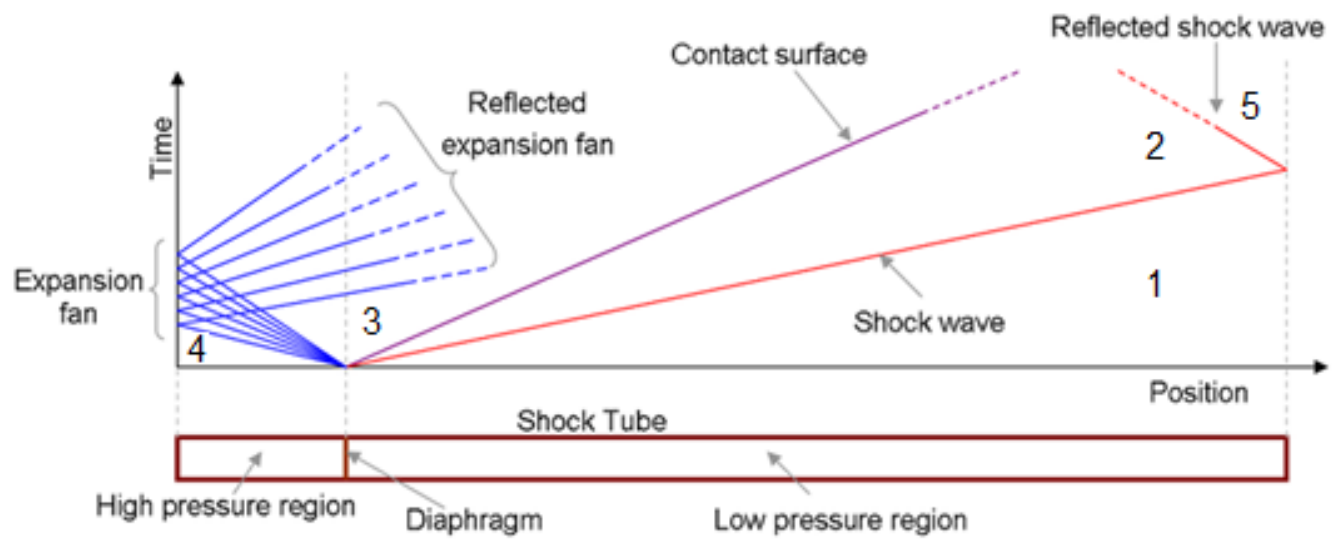

Figure 1. Representative x-t diagram and schematic for reflected shock tube.

\section{Thermochemical Nonequilibrium Modeling}

The presented work will only focus on translational and vibrational nonequilibrium modeling, specifically the vibrational-translational energy transfer process. The V-T process is isolated by investigating cases that are highly dilute mixtures of $\mathrm{O}_{2}-\mathrm{Ar}$. The additional energy modes are assumed to be either in equilibrium (rotational energy mode in equilibrium with translational energy) or frozen (electron/electronic are unactivated). The nonequilibrium between the translational and vibrational energy modes is captured by the use of the two-temperature approach and the Master Equation approach. The models utilize a translational temperature $\left(T_{t}\right)$ and vibrational temperature $\left(T_{v}\right)$ in order to describe the energy of the respective modes. The presented work investigates two levels of fidelity in this portion of the modeling in order to study how these respective temperatures, and thus, energy modes, evolve and interact (standard two-temperature model and state-resolved method).

\section{A. Low-fidelity, Standard two-temperature model}

In the standard two-temperature model, the vibrational nonequilibrium behavior is captured by using the MillikanWhite vibrational relaxation parameter, $\mathrm{p} \tau_{\mathrm{V}}$ (pressure times vibrational relaxation time), and the Landau-Teller relaxation equation. The vibrational relaxation parameter is calculated using the following equation. The equation has two parameters that are unique for particular species collisions, reduced mass $(\mu)$ and the slope parameter (A), and the translational temperature $\left(T_{t}\right)$. The full details of calculating these parameters can be found in the MillikanWhite paper ${ }^{4}$. The calculations for $\mathrm{O}_{2}$ - $\mathrm{Ar}$ in this study use the values of 165.0 and 17.8 for the slope parameter (A) and the reduced mass $(\mu)$ respectively. It should be noted that the population distribution of the vibrational states is implicitly assumed to be Boltzmann in this methodology, and only single-quantum transitions are permissible.

$$
p \tau_{V}=\exp \left[A\left(T_{t}^{-\frac{1}{3}}-0.015 \mu^{\frac{1}{4}}\right)-18.42\right]
$$

American Institute of Aeronautics and Astronautics 
Park ${ }^{1}$ also introduced a high temperature correction term that is added to vibrational relaxation time of MillikanWhite. The correction term is needed to account for the fact that elastic collisions occur and, limit the excitation rate ${ }^{1}$. The correction term has the following form where $\mathrm{n}$ is the total number density and $\mathrm{C}$ is the average thermal speed.

$$
\tau_{\text {Park }}=\frac{1}{n \sigma_{v} C} \quad \sigma_{v}=3 \times 10^{-21}\left(\frac{50,000}{T}\right)^{2}
$$

The vibrational energy evolution is then governed by the calculated vibrational relaxation time through the following Landau-Teller equation. The difference between the current vibrational energy $\left(E_{v}\right)$ and the equilibrium vibrational energy $\left(\mathrm{E}_{\mathrm{v}}{ }^{*}\right)$ drives the evolution.

$$
\frac{d E_{V}}{d t}=\frac{E_{V}^{*}-E_{V}}{\tau_{V}}
$$

Additionally, the dissociation reactions are modeled using the reaction rate coefficient from the Arrhenius equation (shown below). The equation is a function of species properties and the geometrically-averaged temperature $\left(T_{a}=\right.$ $\sqrt{T_{t} T_{v i b}}$ ) that was proposed by Park. Further details can be found in Ref. 2. The current study of $\mathrm{O}_{2}$-Ar uses $1.8 \mathrm{e} 18$ $\mathrm{cm}^{3} / \mathrm{mol},-1.0$, and $59,360 \mathrm{~K}$ for $\mathrm{C}, \mathrm{n}, \theta$ and respectively. The results of this method are referred to as " $2 \mathrm{~T}$ ".

$$
k_{f}=C T_{a}^{n} \exp \left(-\frac{\theta}{T_{a}}\right)
$$

\section{B. High-fidelity, State-resolved method}

The higher level of fidelity in the presented work is the state-resolved method. The vibrational quantum state populations are explicitly tracked and evolved by utilizing a master equation formulation. The state-to-state rates employed in the master equation are evaluated using two different methods. Both models utilize the Dunham expansion to calculate the anharmonic energy levels and consider 37 vibrational quantum levels. The Dunham expansion parameters used in this study are $1580.0 \mathrm{~cm}^{-1}$ and $12.0 \mathrm{~cm}^{-1}$ for $\mathrm{w}_{\mathrm{e}}$ and $\mathrm{w}_{\mathrm{e}} \mathrm{X}_{\mathrm{e}}$.

First, the semiclassical analytical FHO model ${ }^{5-8}$ is applied to calculate the state-to-state rates. The full details of this method can be found in Ref 6. The governing parameter for the FHO model is exponential potential parameter $(\alpha)$. The current study uses 3.82 Angstroms $^{-1}$ for the $\mathrm{O}_{2}$-Ar system. The FHO model allows for multi-quantum transitions. The calculations in this paper limit the multi-quantum transitions to \pm 5 quantum levels. Additionally, the state-resolved, bound-free transition rates (analogous to dissociation rates) are modeled using the modified rates of Esposito9. The results of this method are referred to as "STS-FHO".

Second, the state-to-state rates and bound-free rates are obtained from a detailed QCT analysis performed by Kim and Boyd ${ }^{14}$. The analysis considered all quantum transitions. These results are referred as "STS-QCT", and represent the highest level of fidelity in vibrational nonequilibrium modeling investigated.

The use of a state-resolved method increases the computational cost of the calculations. The state-resolved method exhibits approximately an order of magnitude high computational cost relative to the standard two-temperature approach. This observation is expected based on the number of equations involved in each analysis. The low-fidelity method solves 4 equations ( 3 flow equations and 1 vibrational energy equation), while the state-resolved solves 40 equations (3 flow equations and 37 vibrational master equations).

\section{Flow Modeling}

By ignoring the small boundary layer formed along the walls of the shock tube, the flows are accurately described using the one-dimensional Euler equations, augmented by additional vibrational relaxation equations.

\section{A. Two-Steady Shock Analyses}

The two-steady shock method utilizes reference frame transformations to chain together the incident shock relaxation and reflected shock relaxation in order to represent the reflected shock tube flow. The method inherently assumes uniform 1-D regions. The term uniform in this context refers to the flow conditions that drive the nonequilibrium behavior. This includes the flow conditions before and after a shock wave. In other words, the nonequilibrium behavior is universal within a given region (all particle paths will have the same nonequilibrium 
relaxation in a given region) when this assumption is made. This assumption is limiting in the general sense because the varying incubation times, within say the post incident shock region, will introduce varying flow conditions at the reflected shock. However, for the highly dilute cases that are included in this study, the vibrational mode has little effect on the bulk flow due to the low concentrations of oxygen molecules, and the assumption is rather appropriate. The method also relies on the end wall velocity constraint to calculate the reflected shock strength. The steady shocks are modeled as discontinuities governed by the Rankine-Hugoniot relations. The vibrational mode is assumed to be frozen across the discontinuity.

\section{B. Unsteady Flow Solver}

The unsteady method utilizes a traditional CFD solver ${ }^{10}$ to model the 1-D behavior of the reflected shock tube in space and time. No assumptions are made about the uniformity of the various flow regions, and shock behavior is a byproduct of the simulation, not an input. Figure 2 provides a sample flow field solution obtained with the unsteady CFD flow solver.

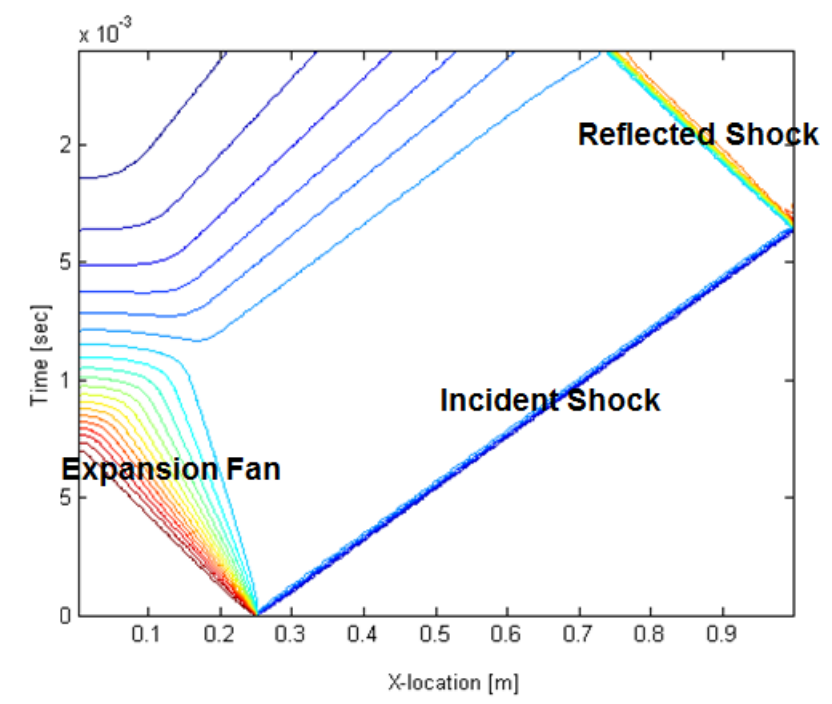

Figure 2. Sample flow field result obtained with the unsteady flow solver

\section{Experimental Data}

The experimental data were obtained from a reflected shock tube facility at Stanford University ${ }^{11}$. The facility utilizes a laser absorption diagnostic for the measurements ${ }^{12}$. The method is utilizing molecular oxygen absorbance. The data collection point was $2 \mathrm{~cm}$ from the end wall of the shock tube.

Since the absorption is the measured quantity in the facility, a model was required to recast the computational flow/thermodynamic quantity results to and from absorption for direct comparison. The full details of the model can be found in Ref. 13.

\section{Computational Results and Comparisons}

\section{A. Test Case 1}

The test case 1 conditions are chosen to all a focus on vibrational nonequilibrium. The mole fraction composition is $98 \%$ Argon and $2 \% \mathrm{O}_{2}$. The incident shock flow conditions are $\mathrm{T}_{1}=298 \mathrm{~K}, \mathrm{P}_{1}=7.125$ torr, $\mathrm{M}_{\mathrm{s}}=3.57$. Figure 3 shows profiles of the translational and vibrational temperatures. Note that these quantities for the experimental data are deduced by utilizing an iterative method that matches the temperatures to the measured absorption by applying the absorption model described in Ref. 13 with the measured pressure at the data collection point. The profiles show the passage of the incident shock ( -165 microsec) and the subsequent vibrational relaxation (from -165 to 0 microsec). The convention of defining $t=0$ at the passage of the reflected shock has been adopted ${ }^{11}$. The vibrational relaxation

American Institute of Aeronautics and Astronautics 
after the reflected shock reaches equilibrium in approximately 50 microsec. The results reveal that all models agree very well with each other and the experimental data. This result is expected since the case is at relatively mild temperatures. The assumptions that may hinder the low-fidelity modeling are still valid for the test case flow conditions. Namely, Figure 5 shows that the vibrational population distribution remains Boltzmann during the reflected shock vibrational relaxation. It is also found that the multi-quantum transition rates are all at least an order of magnitude less than the single-quantum transition rates, and most being multiple orders of magnitude less. These findings indicate that the assumptions of the $2 \mathrm{~T}$ approach are adequate for this relatively mild condition.

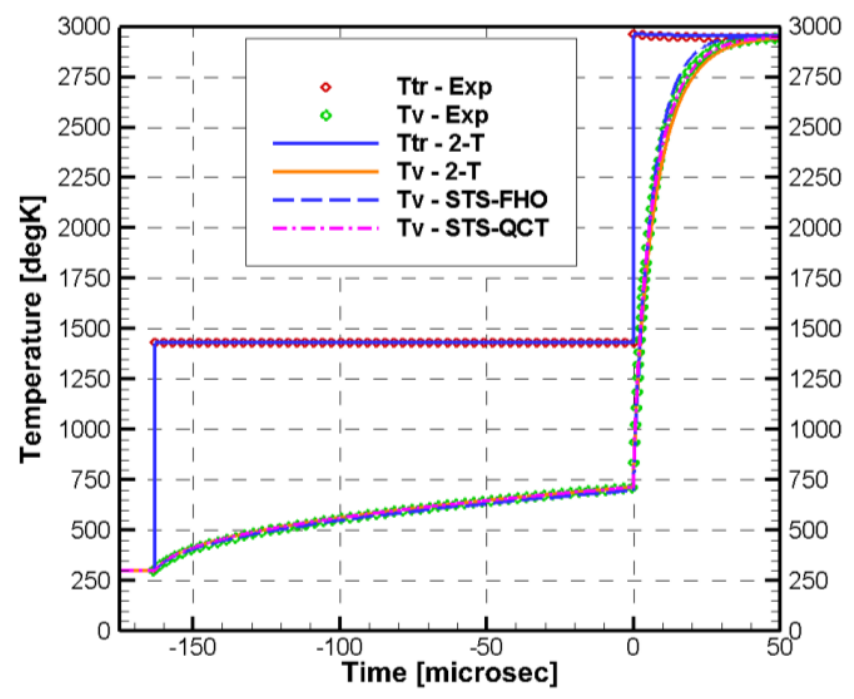

Figure 3. Temperature profiles obtained with different relaxation models for test case 1 $T_{1}=298 K, P_{1}=7.125$ torr, $M_{s}=3.57$ (incident shock)

Figure 4 compares the vibrational state population distributions obtained directly in STS-FHO to the Boltzmann distribution that is implied by vibrational temperature solutions obtained in the 2-T approach. It is interesting to note that the STS-FHO distribution deviates from the Boltzmann distribution at higher vibrational levels during the post reflected shock relaxation before it reaches equilibrium. The temperature profiles do not show significant differences that suggests these deviations can be considered small in the sense that they do not have much influence on the overall solution. This notion can be rationalized by the fact that the deviations occur at high vibrational quantum states which have very low populations and the deviations are all less than an order of magnitude.

American Institute of Aeronautics and Astronautics 


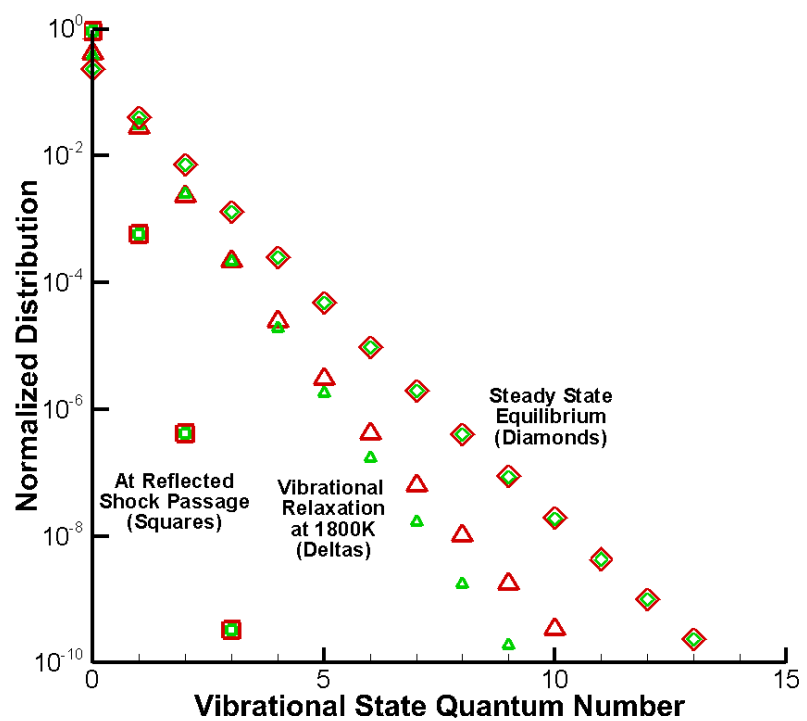

Figure 4. Comparison of normalized vibrational distributions for test case 1 Small symbols - Boltzmann distribution from 2T; Large symbols - STS-FHO

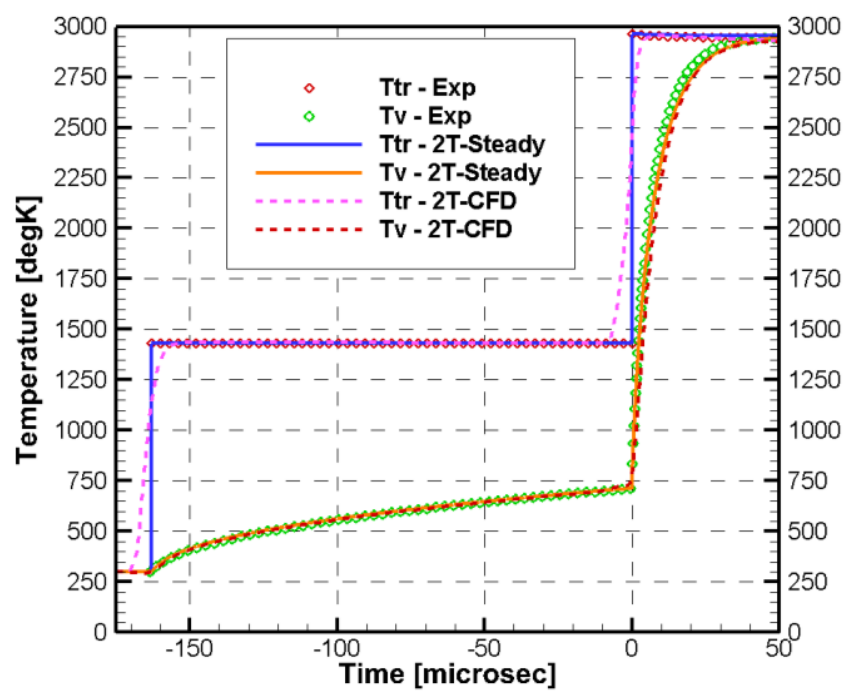

Figure 5. Temperature profiles obtained with different flow solvers for test case 1 $T_{1}=298 K, P_{1}=7.125$ torr, $M_{s}=3.57$ (incident shock)

Figure 5 shows a comparison between the two different flow modeling methods utilizing the same vibrational nonequilibrium modeling (2T). The results agree very well with each other. The only noticeable difference is seen in the translational temperature for the unsteady CFD method. The translational temperature profile shows slight shock smearing around the incident and reflected shocks. The smearing is an artifact of the numerical method. The discontinuity can be more sharply captured by expending more computational effort, however the smearing has no effect on the vibrational relaxation that is the main focus of this study.

American Institute of Aeronautics and Astronautics 


\section{B. Test Case 2}

A stronger case is evaluated using the various vibrational nonequilibrium models. The flow composition involves a mole fraction of $99.9 \%$ argon and $0.1 \% \mathrm{O}_{2}$. The flow conditions are $\mathrm{T}_{1}=295 \mathrm{~K}, \mathrm{P}_{1}=1.0$ torr, $\mathrm{M}_{\mathrm{s}}=5.9$. The incident portion of the relaxation has essentially no dissociation so for clarity, this portion of the profiles is omitted to better focus on the reflected shock relaxation. Figure 6 shows the temperature behavior following the reflected shock (note there is no experimental data available for this case). The case is strong enough to produce the quasi-steady state (QSS) behavior in which the vibrational energy input (VT process) and the vibrational energy output (dissociation) are equal. This is the reason for the constant vibrational temperature part way through the relaxation process. Under these conditions, there are clear differences between the results obtained with each of the modeling methods.

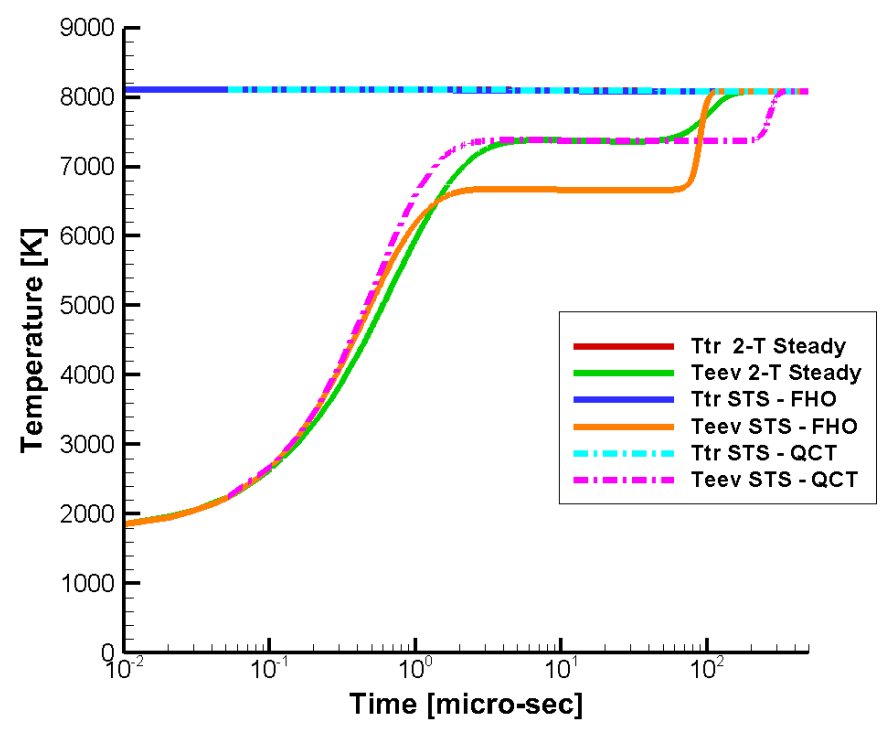

Figure 6. Temperature profiles obtained with different relaxation models for test case 2 $T_{1}=295 K, P_{1}=1.0$ torr, $M_{s}=5.9$ (incident shock)

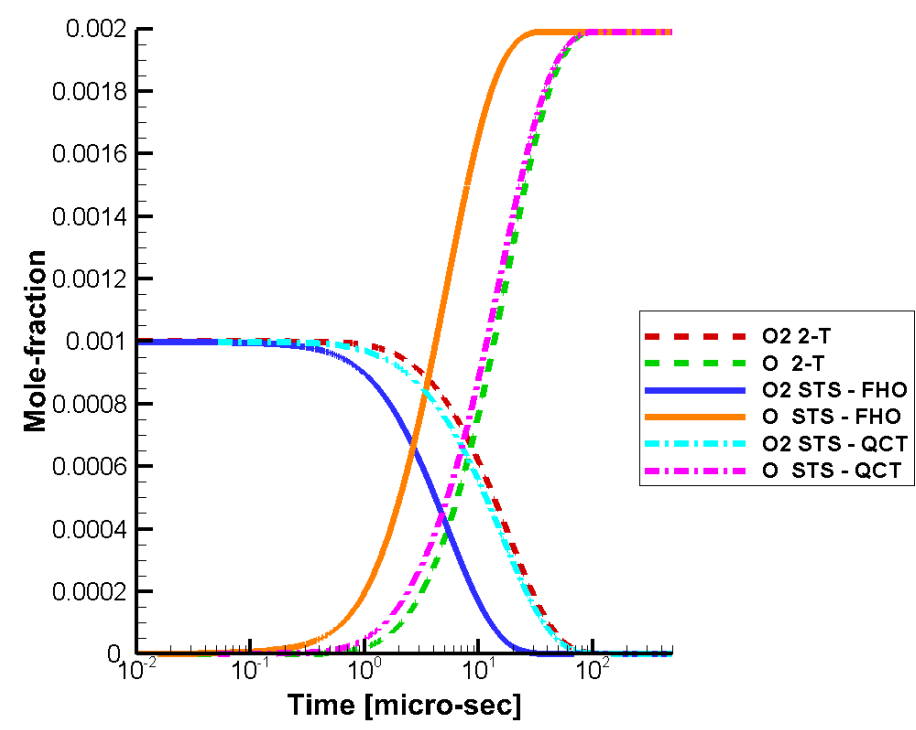

Figure 7. Oxygen mole-fraction profiles obtained with different relaxation models for test case 2 $T_{1}=295 K, P_{1}=1.0$ torr, $M_{s}=5.9$ (incident shock)

American Institute of Aeronautics and Astronautics 
Profiles of the mole fraction of the oxygen species after the reflected shock passage are shown in Figure 7 . The results for test case 2 show that vibrational nonequilibrium modeling fidelity does matter for these stronger conditions. Each method reaches equilibrium at different times. These relaxation differences may be critical for hypersonic vehicle design.

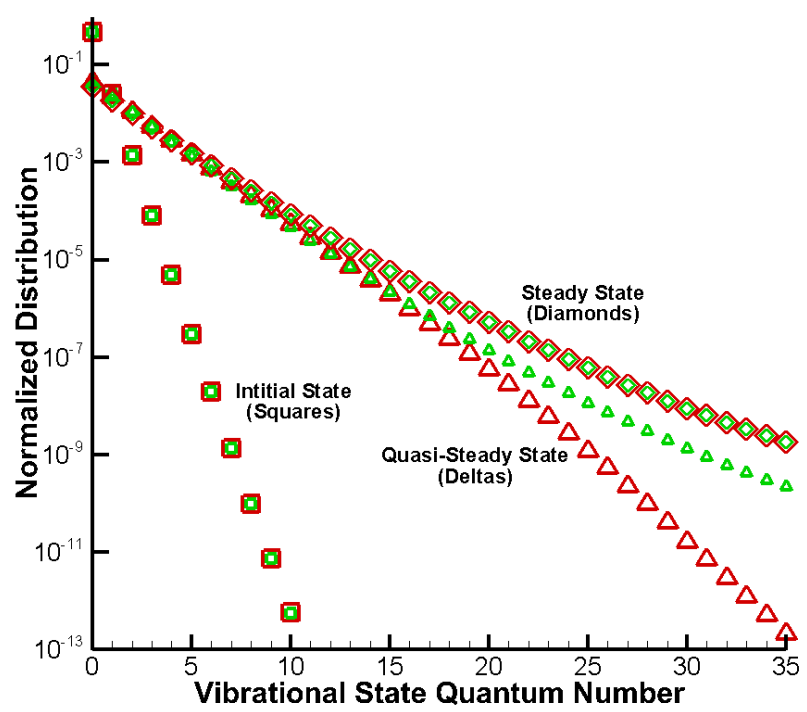

Figure 8. Vibrational population distribution comparison for test case 1
Small symbols - Boltzmann distribution; Large symbols - STS-FHO

Figure 8 compares the vibrational state population distributions obtained directly from STS-FHO and the Boltzmann distribution that is implied by the the vibrational temperature solution from the 2-T approach. The STSFHO result deviates from the Boltzmann distribution during the QSS phase of the relaxation process. The distribution deviations are an order of magnitude or more for some of the high vibrational states. It has been shown that these two models produce very different temperature profiles. The conditions of this test case are stronger and involve a large amount of dissociation. The next section aims to better understand the effects of the vibrational modeling differences and dissociation modeling differences.

Figure 9 compares the 2T results for the two-steady shock approach and the unsteady CFD approach. Similar to the previous test case, there is little difference between the results for the two different solvers. This result affirms the notion that the uniformity assumption is applicable for these highly diluted cases. It is antipated that cases that are not highly diluted will not see this level of agreement. Higher concentrations of molecular oxygen would cause the thermodynamic bulk flow quantities to be affected by the vibrational energy mode behavior. This effect would be most apparent at the reflected shock. The post-incident shock flow is not subjected to the same incubation time during the relaxation process. Flow near the wall undergoes post-incident shock relaxation for a shorter amount of time in comparison to a location further from the wall. This variation in incubation time produces a variation in the vibrational conditions at the time that the reflected shock passes back into the flow. For such non-dilute cases, this means that the bulk flow thermodynamic variables will vary and produce variation in the shock strength of the reflected shock. In a space-time sense, this will be evident by the observation of a curved reflected shock and nonuniform conditions in the post-reflected shock region. This behavior will violate the assumptions made in the twosteady shock analysis method. Further investigations are needed to quantify this modeling impact.

American Institute of Aeronautics and Astronautics 


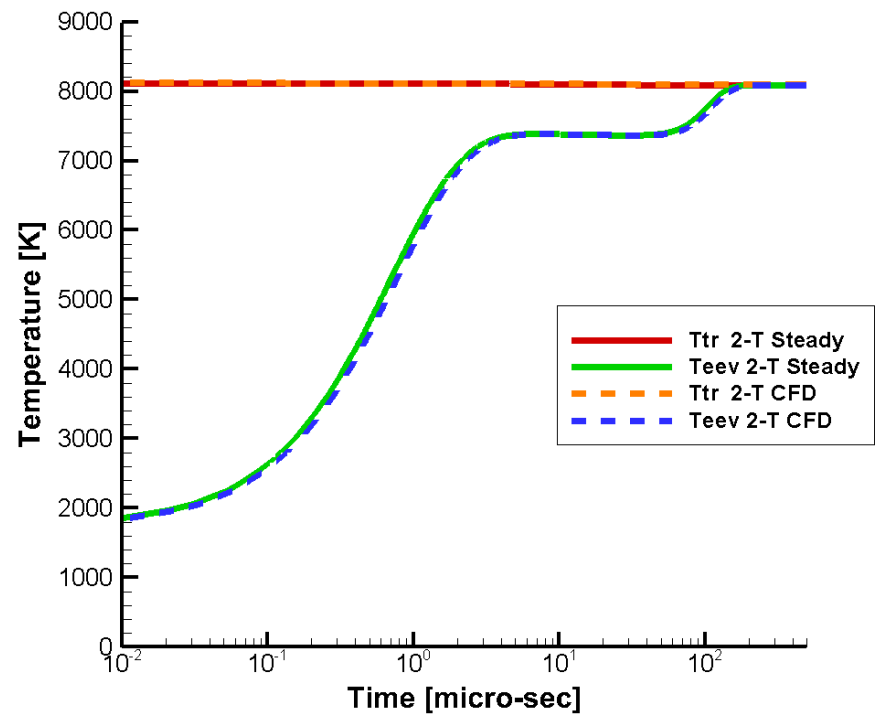

Figure 9. Temperature profiles obtained with different flow solvers for test case 2 $T_{1}=295 K, P_{1}=1.0$ torr, $M_{s}=5.9$ (incident shock)

\section{Detailed Rate Comparison}

It has been shown that the models produce different results for stronger shock conditions. This section aims to investigate the differences in the vibrational and chemical modeling in order to provide an explanation for these differences.

Figure 10 compares the vibrational relaxation parameter for each of the models employed in this study. The vibrational relaxation parameter is the output of the Millikan-White curve fit, but must be calculated for the stateresolved approaches. The calculation of the vibrational relaxation parameter is performed through a master equation analysis using the state-to-state rates. The master equation analysis gives the evolution of vibrational energy over time. This information can then be recast as a relaxation parameter through the use of the Landau-Teller equation. As would be expected, the vibrational relaxation parameter values are very similar for lower temperatures where all of the assumptions for the models are valid. As the temperature increases (to the left), the rates obtained by the various models begin to deviate, and this is due to some of the assumptions becoming invalid. For example, around 4500K, multi-quantum transitions become more prevalent. The assumption of single-quantum transitions within the LandauTeller formulation using the Millikin-White rates causes those results to begin deviating from the actual physics. The Millikin-White rates that are used in the 2-T model are shown in two forms. First is the original Millikin-White rates. The second version includes the high temperature correction that was suggested by Park ${ }^{1}$. The correction has proven to be effective for other species interactions. However, the standard correction appears to not be adequate for the $\mathrm{O}_{2-}$ Ar interaction. Since this is an empirically based correction, there may be an opportunity to develop a better correction factor for this system. The STS-QCT model is considered the highest fidelity, and thus most accurate model investigated.

American Institute of Aeronautics and Astronautics 


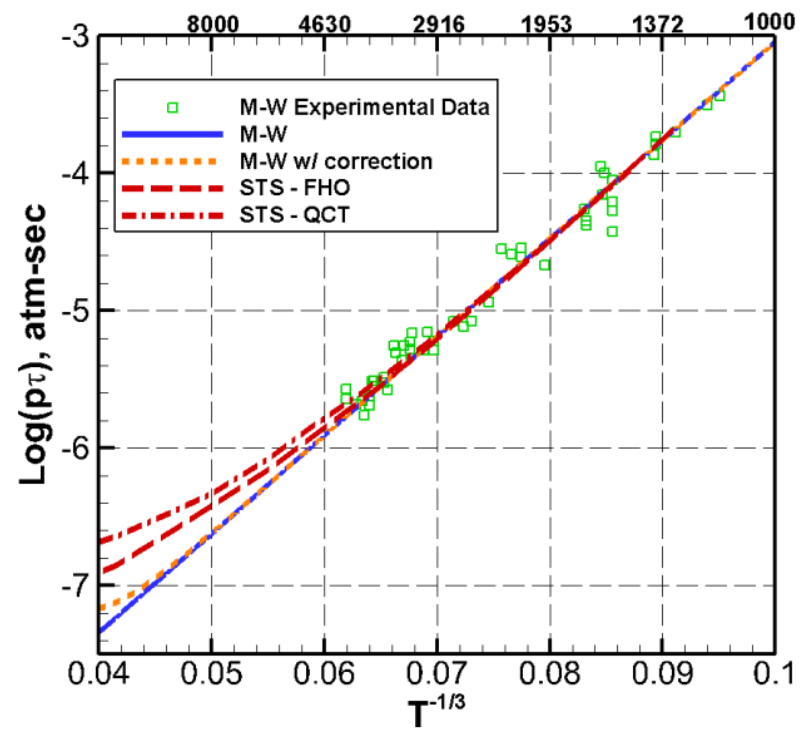

Figure 10. Vibrational relaxation parameter as a function of temperature

In concert with the VT transfer process, it is also necessary to consider the vibrational energy loss due to dissociation. Figure 11 compares the rates of vibrational energy lost through dissociation as predicted by each of the methods for the test case 2 in which a significant amount of composition change occurs.

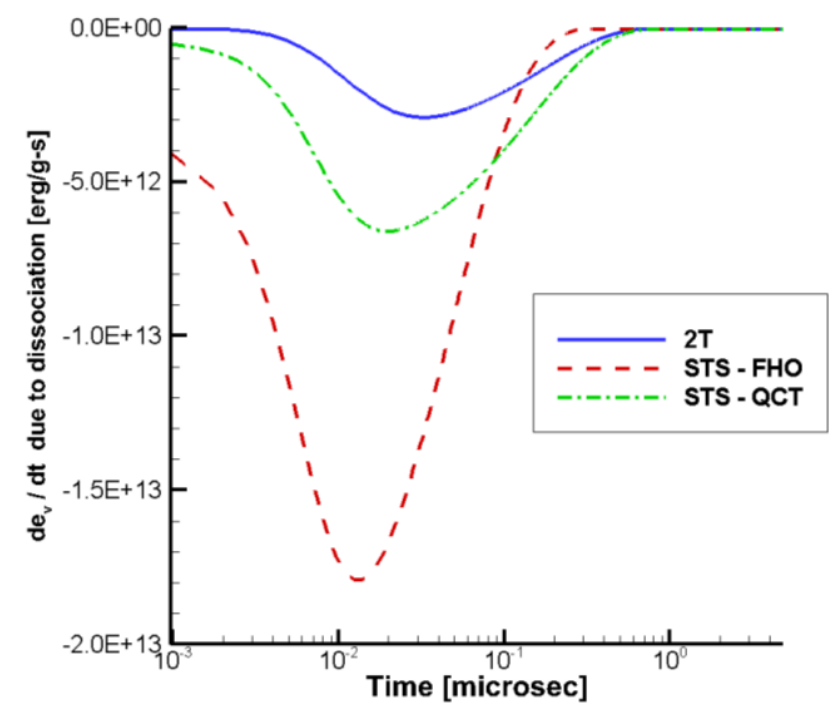

Figure 11. Vibrational energy lost through dissociation (Test Case 2)

The profile from the STS-FHO model stands out as predicting a significantly higher rate of energy removal compared to the other models. This behavior explains the difference in vibrational temperature profiles seen in Figure 6 in which STS-FHO predicts lower Tv in the QSS period than the other models. However, the vibrational relaxation parameters agree within 10\% for the STS-QCT and STS-FHO at this temperature as shown in Fig. 10. This suggests that the vastly different QSS behavior arises not from the vibrational relaxation modeling, but rather from differences in the bound-free rates. Figure 12 shows the equilibrium dissociation rates for the various models for further comparison. At the Test Case 2 conditions, the QCT rate is a factor of 2 and the FHO (Esposito) rate is almost a full order of magnitude larger than the $2 \mathrm{~T}$ (Arrhenius) rate. This supports the notion that the difference in QSS behavior for Test Case 2 is driven primarily by the differences in the bound-free rate behavior of the models. The accuracy of these dissociation rates is not well known for the $\mathrm{O}_{2}$ - $\mathrm{Ar}$ system.

American Institute of Aeronautics and Astronautics 


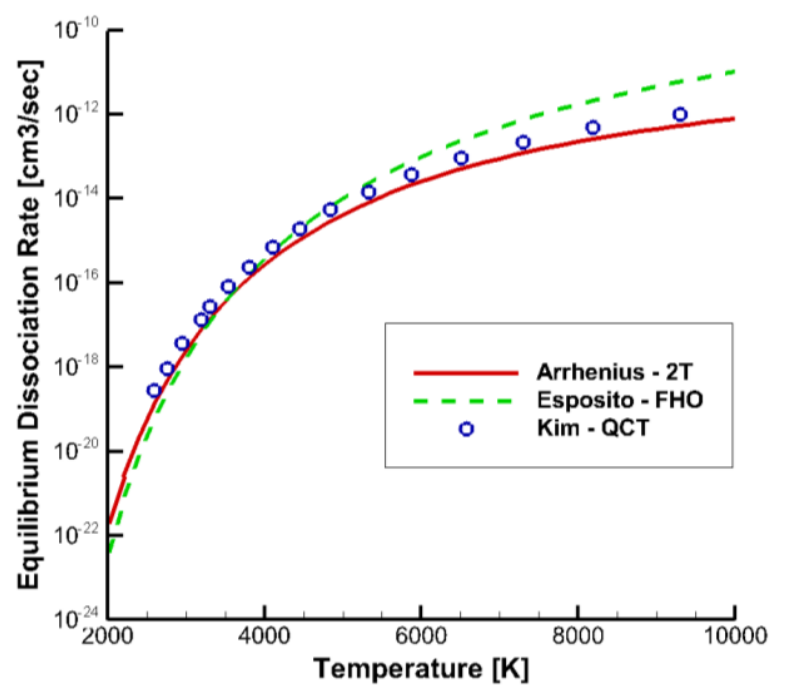

Figure 12. Equilibrium dissociation rate as a function of temperature

\section{Summary and Conclusions}

In the present study, three different levels of fidelity in vibrational nonequilibrium modeling were investigated. The three levels of fidelity are the two temperature model, state-resolved Master Equation with FHO state-to-state rates and Esposito bound-free rates, and state-resolved Master Equation employing QCT state-to-state and bound-free rates. The results show that all levels of fidelity are adequate for mild shock conditions (roughly < $4500 \mathrm{~K}$ ). However, at stronger shock conditions, signicant differences are found in the results obtained with lower and higher fidelity modeling. Additional comparisons with measured data under such conditions is required to evaluate the relative merits of the various models. It is anticipated that state-resolved modeling is needed, but the choice of state-to-state rates must be determined. The presented results show that the pure vibrational relaxation behavior is similar for FHO and QCT state-to-state rates. Additionally, no appreciable difference between the two-steady shock analyses and the CFD solver was observed for the flow modeling. This result is understandable for highly dilutes cases like the ones studied in this paper. However, further investigation into cases that are not highly diluted is needed. Also, the state-resolved method exhibits aprroximately an order of magnitude higher computational cost compared to the standard twotemperature approach.

\section{Acknowledgments}

The authors thank Stanford University for valuable discussions about the experimental details and the sharing of the absorption model. The authors gratefully acknowledge funding for this work through Air Force Office of Scientific Research Grant FA9550-12-1-0483.

\section{References}

${ }^{1}$ Park, C., "Review of Chemical-Kinetic Problems of Future NASA Missions I: Earth Entries," Journal of Thermophysics and Heat Transfer, Vol. 7, No. 3, 1993, pp. 385-398.

${ }^{2}$ Park, C., "Two-Temperature Interpretation of Dissociation Rate Data for $\mathrm{N}_{2}$ and $\mathrm{O}_{2}, "$ AIAA Aerospace Sciences Meeting, AIAA-88-0458, 1988.

${ }^{3}$ Kim, J.G. and Boyd I.D., "State-resolved Master Equation Analysis of Thermochemical Nonequilibrium of Nitrogen," Chemical Physics, Vol. 415, 2013, pp.237-246.

American Institute of Aeronautics and Astronautics 
${ }^{4}$ Millikan, R.C. and White, D.R., "Systematics of Vibrational Relaxation,” Journal of Chemical Physics, Vol. 39, No. 12, 1963, pp. 3209-3213.

${ }^{5}$ Adamovich, I.V. and Rich, J.W., "Three -dimensional nonperturbative analytical model of vibrational energy transfer in atom-molecule collisions," Journal of Chemical Physics, Vol. 109, No. 18, 1998, pp. 7711-7724.

${ }^{6}$ Adamovich, I.V., Macheret, S.O., Rich, J.W., and Treanor, C.E., "Vibrational Relaxation and Dissociation Behind Shock Waves Part 2: Master Equation Modeling," Journal of Thermophysics and Heat Transfer, Vol. 33, No. 6, 1995, pp. 1070-1075.

${ }^{7}$ Adamovich, I.V., "Three-dimensional Analytic Model of Vibrational Energy Transfer in Molecule-Molecule Collisions," AIAA Journal, Vol. 39, No. 10, 2001, pp. 1916-1925.

${ }^{8}$ Billings, G.D. and Kolesnick, R.E., "Vibrational Relaxation of Oxygen. State to State Rate Constants" Chemical Physics Letters, Vol. 200, No. 4, 1992, pp. 382-386.

${ }^{9}$ Esposito, F., Armenise, I., Capitta, G., Capitelli, M., "O-O2 state to state vibrational relaxation and dissociation rates based on quasiclassical calculations" Chemical Physics.

${ }^{10}$ Scalabrin, L.C. and Boyd, I.D., "Development of an Unstructured Navier-Stokes Solver for Hypersonic Nonequilibrium Aerothermodynamics," AIAA Paper 2005-5203, 2005.

${ }^{11}$ Owen, K., Davidson, D.F., and Hanson, R.K., Private Communication.

${ }^{12}$ Chang, H.A., Baer, D.S., and Hanson, R.K., "Semiconductor Laser Diagnostics of Oxygen for Hypersonic Flowfield Measurements," AIAA Paper 93-0628, January 1993.

${ }^{13}$ Bykova, N.G., Kuznetsova, L.A., "Study of the absorption characteristics of molecular oxygen in the Schumann-Runge system at high temperatures" SpringerLink Optics and Spectroscopy, Vol. 105, No. 5, 2008, pp. 668-673.

${ }^{14}$ Kim, J.G., and Boyd, I.D., "Thermochemical Nonequilibrium Modeling of Electronically Excited Molecular Oxygen,” AIAA Thermophysics Conference Paper TBD, 2014.

American Institute of Aeronautics and Astronautics 